\title{
Pervasive Patient Timeline for Intensive Care Units
}

\author{
André Braga ${ }^{1}$, Filipe Portela ${ }^{1,2}$, Manuel F. Santos ${ }^{1}$, José Machado ${ }^{1}$, António Abelha ${ }^{1}$, \\ Álvaro Silva ${ }^{3}$, Fernando Rua ${ }^{3}$ \\ ${ }^{1}$ Algoritmi Research Centre, University of Minho, Portugal \\ ${ }^{2}$ ESEIG, Porto Polytechnic, Porto, Portugal \\ ${ }^{3}$ Intensive Care Unit, Centro Hospitalar do Porto, Porto, Portugal \\ andre.nevoabraga@gmail.com, \{cfp, mfs\}@dsi.uminho.pt, \{jmac, \\ abelha\} @di. uminho.pt \\ moreirasilva@me.com; fernandorua.sci@chporto.min-saude.pt
}

\begin{abstract}
This research work explores a new way of presenting and representing information about patients in critical care, which is the use of a timeline to display information. This is accomplished with the development of an interactive Pervasive Patient Timeline able to give to the intensivists an access in real-time to an environment containing patients clinical information from the moment in which the patients are admitted in the Intensive Care Unit (ICU) until their discharge This solution allows the intensivists to analyse data regarding vital signs, medication, exams, data mining predictions, among others. Due to the pervasive features, intensivists can have access to the timeline anywhere and anytime, allowing them to make decisions when they need to be made. This platform is patient-centred and is prepared to support the decision process allowing the intensivists to provide better care to patients due the inclusion of clinical forecasts.
\end{abstract}

Keywords. Pervasive Patient Timeline, Intensive Medicine, Intensive Care Unit, INTCare, Patient-centred, Timeline

\section{Introduction}

Over the years, technological devices and information systems have been introduced into medicine with the purpose of increasing the quality and efficiency of the health care facilities in the patient best interest [1,2].

In the Intensive Medicine field several medical equipment was introduced into the Intensive Care Units (ICU) resulting in an increase of the amount of data available [3] (e.g. ventilators, vital signs monitors) in real-time. The high number of data available can be a problem because they also can be overwhelming to deal with, hindering their ability to combine all the information, analyse it and make a decision [4].

Another important aspect is also the fact that almost all of the device presents the information in its own unique way, be it graphs, tables, text or any other format, resulting in a need of learning about how to interpret each one of the devices.

These reasons gave origin to this research work. In this work a new and interactive platform was developed. It holds in one place data from different data sources 
(interoperability), homogenizes its representation and sorts it chronologically in order to simplify data readability and facilitate the understanding of possible cause-effect relations. Therefore the work focus is to help intensivists in the decision making process. The research project was conducted and implemented in the ICU of the Hospital de Santo António, Centro Hospitalar do Porto.

The Pervasive Patient Timeline is a web platform and it presents several characteristics, such as, real-time data, flexibility, adaptability, interactivity, scalability and one of most important, the pervasive access to its features and the context awareness. With the implementation of these characteristics it focuses on addressing the difficulty associated with dealing with too many information presented in different ways by different devices. The Pervasive Patient Timeline looks to sort chronologically and standardize the presentation of clinical information in one platform by gathering the data from various medical devices and presenting it in its interface. In this way intensivists can have a faster and easier access to all the information that they need to make decisions.

Apart from this introduction, the article consists of other five sections. The second section is the Background focused on contextualizing of the work. It is divided in four sub-sections, such as Intensive Care, the INTCare, the Timeline and Pervasive Healthcare. In section three is presented the research methodology used for the development of this research. The next section talks about the Pervasive Patient Timeline, its development and features. The fifth section discusses the work developed and the results obtained, while the last one, Conclusion, makes final remarks on what was achieved and the importance of the research done.

\section{Background}

\subsection{Intensive Care}

In field of Medicine there is a branch called Intensive Medicine (IM) which it is responsible for treating patients in serious health conditions and recover them to a health state and quality of life prior to the conditions affecting them [5]. An Intensive Care Unit (ICU) is a critical environment where patients are usually in coma and they are always being monitored [6]. The life support is done using many technical devices in order to ensure that the patients can get back to the previous health state [7]. These devices, like bedside monitors collect and present patients' data in real-time allowing intensivists to have a better understanding of the patients' condition.

\subsection{INTCare}

The INTCare system is a Pervasive Decision Support System (PDSS) developed as part of a project with the same name. It is responsible for modernizing the information system of the Intensive Care Unit of the Hospital de Santo António, Centro Hospitalar do Porto (CHP). The system acts autonomously and in real-time, and it is composed by four subsystems that interact between each other through intelligent agents: Data Acquisition, Knowledge Management, Inference and Interface [6, 8]. 
The purpose of the INTCare system is to help intensivists in the decision making process, by providing capabilities such as monitoring patients' conditions, predict clinical events, like organ failure [9], length of stay [10], readmission [11], among others, and issue alert messages when the patients being monitored have vital signs outside of the normal range [6]. In this way it is ensured that the intensivists have an easier job but also the patients have a better and safer care. The new knowledge that the system provides helps the decision making process

The Pervasive Patient Timeline was developed under the phase II of the INTCare project, therefore it is going to be integrated with the existing INTCare system, taking advantage of its features.

\subsection{The Timeline}

The timeline is one of many common ways of representing graphically information. Its most characteristic feature, which distinguishes it from others, is the fact that it displays its content sorted chronologically.

The representation of information in a timeline format allows to observe when certain events happened, the duration of the event and the time between the events, overlaps between events, among others. The granularity of the timeline is also an important part of its design, because it will define the level of detail we want for it.

Historically, timelines have always been used to catalogue events of various types, such as, wars, diseases, revolutions, discoveries, epidemics, among others [12]. In the field of medicine they are commonly used in records concerning diseases outbreaks, studies, but also on the daily practice. In Intensive Medicine they are also presented in the monitoring of blood pressure, heart rate, among others [13].

Nowadays with the advances in technology it is possible to visualize and interact with timelines digitally, allowing to change content without having to redo the timeline, with no space limitations, adding categories to the content displayed, among others [14]. Finally the representation of information using timelines allows the observer to see the data sorted chronologically, understand better the relationships between data, especially cause and effect situations.

\subsection{Pervasive HealthCare}

Pervasive computing is an emerging approach focused on developing intelligent environments where the devices are interconnect and inserted in an environment that offers a continuous, reliable, non-intrusive connectivity with added value. The result of this systems tends to be the improvement of human experience and quality of life without an explicit perception of the underlying interaction between technology [15].

Over the years, technology has evolved towards more pervasive and ubiquitous infrastructures as a result of the increased ease of access to the Internet. As an example of this is the increasing number of mobile devices that are capable of collecting, sharing, storing information [16], but also wireless networks and communications systems [17]. 
The application of these type of systems and devices on the medicine field makes it possible to create a pervasive healthcare environment where everyone has access to healthcare anywhere and anytime, as a result of the removal of time and location restrictions [17].

Therefore pervasive data can also be seen as data that can be accessed anytime and anywhere. In critical environments, such as Intensive Care Units, where the decisions need to be made at the right time, the complete information needed to make an assessment is not always available there. Being not possible to overcome all medical errors, the pervasive data approaches can facilitate the data distribution. Healthcare quality can be enhanced through the reduction of information redundancy and allowing it to be stored in mobile devices or in situated devices available in the locations where decisions will be made [18]. An example of pervasive data is the system which sent alerts from the monitoring patient system. These alerts are sent when patients are out of the normal range of the attributes monitored.

In general pervasive healthcare helps healthcare professionals by creating an environment allowing them to access information anywhere and anytime.

\section{Research Methodology}

The development of this research was done under the Design Science Research (DSR) methodology, in order to ensure an appropriate orientation for the research life cycle. The DSR consists in a set of analysis techniques and perspectives use in research in Information Systems. According to Peffers [19], the DSR is composed by six activities: Identify Problem and Motivate, Define Objectives of a Solution, Design and Development, Demonstration, Evaluation and Communication.

In the context of the research, the iteration process had a beginning centred on the problem, having started in the moment of problem definition and its importance. The first activity, Identify Problem and Motivation, is focused in understanding how the implementation of a pervasive timeline could improve the decision making process. In the second activity, Define Objectives of a Solution, the objective of the research was established in which it is the development of a pervasive timeline capable of providing new knowledge and facilitate the decision making process in clinical environments, without space and time restrictions. The third activity, Design and Development, consisted in executing tasks with the purpose of designing the characteristics of the artefact, followed right after by the development of the artefact itself. In the fourth activity, Demonstration, clinical data of patients were incorporated into the pervasive timeline, as a way to simulate and present the disposition of the information in the timeline. The fifth activity, Evaluation, a set of metrics responsible for the evaluation of the Pervasive Patient Timeline performance in the clinical environment was defined. Lastly, in the six activity, Communication, some scientific papers were written and published in journals and conferences, in order to express the importance of this work. 


\section{Pervasive Patient Timeline}

The Pervasive Patient Timeline can be seen as a Clinical Decision Support System (CDSS) having as main objective to support the decision making process of intensivists in clinical environments. It can be done by centralizing data from various data sources making it interoperable and presenting it in a more standardized, concise and easy way to understand. The platform works in real time, without location restrictions and it allows to observe the clinical history of patients from the moment they were admitted into the Intensive Care Unit until their discharge. Therefore it is possible to access to past and present information, but also future predictions through the incorporation of Data Mining models' results into the timeline. The data presented in the timeline is acquired by the INTCare system in real-time and treated through the application of an Extract Transforming and Load (ETL) process to ensure the quality of the information acquired.

\subsection{Data Source}

The clinical data used for the development of the Pervasive Patient Timeline was provided by the Intensive Care Unit (ICU) of the Hospital de Santo António, Centro Hospitalar do Porto (CHP). The data is retrieved from six different data sources:

- Vital Signs Monitor (VS) - this source is where patients' vital signs, such as, temperature, heart rate, blood pressure and many others are measured and presented;

- Laboratory $(\mathrm{LAB})$ - the laboratory is responsible for holding the results of patients' exams, like potassium, sodium, leucocytes, urea, among others;

- Drugs System (DS) - this system is responsible for recording the therapeutic plans prescribed to the patients;

- Ventilation Monitor (VM) - in the ventilation monitors are collected data relative inspired oxygen, pulmonary compliance, positive end-expiratory pressure, among others;

- Electronic Nursing Record (ENR) - in this data source are presented all the clinical data validated about patients and human registered;

- Electronic Health Record (EHR) - here are kept the data concerning patients' admission and discharge, chronic diseases and such information that describe the medical history of the patients.

\subsection{Requirements and Features}

The development of this Pervasive Patient Timeline underwent a process of trying existent timeline software and then change and adapt them to the needs of the clinical environment, in order to fulfil the research objective. The choice process was based on an analysis conducted on each of the experimented timelines. The timelines were evaluated based on their features, interface, documentation, development time and extra functionalities. The timeline which was scored highest was selected as the one to use as a base of development. For the development the XAMPP platform was used 
along with the programming languages, PHP and JavaScript. Oracle SQL was used to treat the data before adding it to the timeline. By the end of the development process the following features were implemented:

\section{Data:}

- Possibility of loading into the timeline data retrieved from databases of different data sources.

\section{Granularity:}

- Maximum: 15 to 15 min;

- Minimum: Decade to Decade.

Usability:

- Choose a patient from a list to visualize his data in the timeline;

- Choose the type of data to visualize: all data or only critical data;

- Slide horizontally the timeline, in to visualize past and future events;

- While visualizing the timeline, zoom in and zoom out the granularity;

- Filter the categories of data visible in the timeline;

- Adjust the events time according to the time zone;

- Find events by searching for words in title or description;

- Click to go to the next or previous event;

- Define a date and advance to the defined moment.

\section{Event Features:}

- Title, absolute values of the first hour and maximum, minimum and average hour values;

- Event description, which can contain text, images, links;

- Events with start and end date;

- Capacity to add events to different categories;

- Indicator called importance level that defines the granularity at which the events can be seen (can be important when there are too many events cluttering the timeline).

Since the Pervasive Patient Timeline is a platform, it has some system requirements that are needed for it to function properly:

- Internet access;

- Interoperability with different systems;

- Context awareness;

- Privacy of access to data presented;

- Continuous access to data;

- Database infrastructure.

As main characteristics of the Pervasive Patient Timeline it is the fact of it works in real-time by presented the data collected in the moment; it is flexible and adaptive, allowing the possibility to add new different events to the timeline (e.g. other data sources, data mining models); it is scalable since it allows the increase of data present in its system; its pervasive aspect makes it possible to access the data from the timeline in any location and anytime and lastly, its interactivity provides a more engaging way of data visualization. 


\subsection{Timeline Interface}

In Figure 1, is an overview of the Pervasive Patient Timeline. In this figure is data concerning a patient admitted into an ICU. Specifically, there is information retrieved from the hospital's VS, VM, LAB, DS, EHR and ENR data sources. In this figure is possible to consult critical events (as red), patient admission information and choosing the data sources. As example of event description of an event is possible consult maximum, minimum and average values of the hour, along with the respective unit.

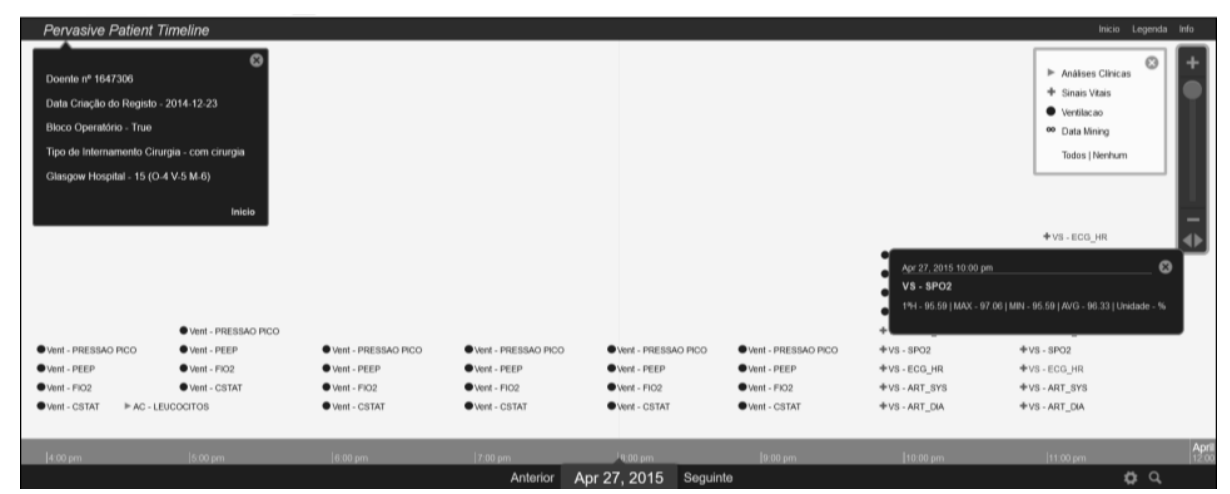

Fig. 1. Example of the Pervasive Patient Timeline

Data mining (DM) predictions which provide the estimated probability for the event that the data mining model covers, were also added to the timeline (Figure 2). With this feature intensivists can start planning ahead before a predicted event occurs. This feature can help reducing costs and manage resources. In this case Data Mining models were developed to predict the vasopressors need [20]. The figure is presenting the probability of a patient need vasopressors in the next hour. Other DM models can be added to the timeline as is predict critical events [21, 22], length of stay [23], outcome, organ failure, sepsis [24] among others,

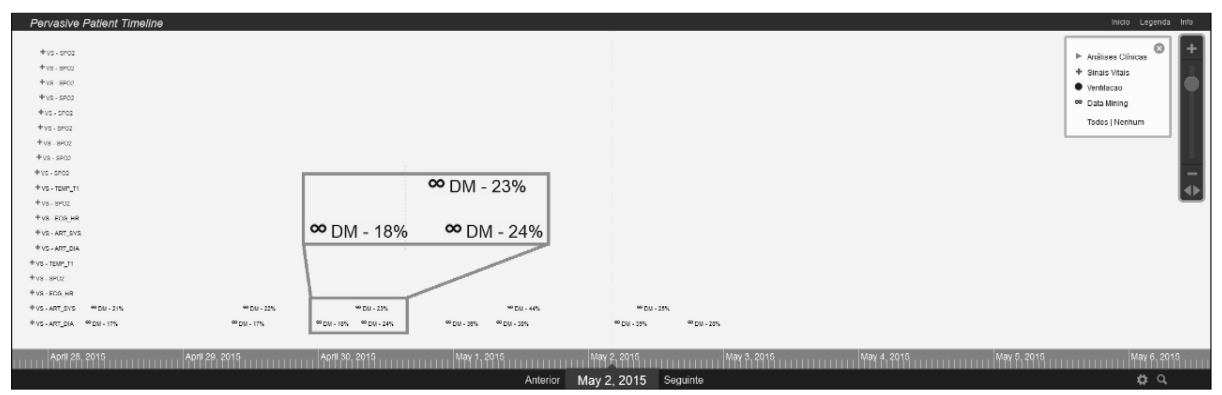

Fig. 2. Data Mining Models in the Pervasive Patient Timeline 


\section{Discussion}

The use of the Pervasive Patient Timeline intends to provide new knowledge in clinical environments, by allowing intensivists to visualize information aggregated from various data sources in only one place and in an innovative way, i.e., the timeline. Here intensivists can access the information in real-time and without place restrictions, allowing them to consult the data when the need arises. The presentation of data sorted chronologically is another of its main features that eases the understanding of cause-effect relations between data and the finding of patterns, which it is fundamental in a critical environment such is Intensive Care Units.

Its interface provides an easy and intuitive control over the information in the timeline. The Pervasive Patient Timeline holds information related to vital sign monitors, laboratory exams, drugs, ventilation monitors, electronic nurse record and electronic health record. It allows to see past historic data, current data and future predictions by using data mining models to make such predictions. The data presented is part of the INTCare system, so the autonomous agents perform ETL tasks in order to provide data with quality. This task is important because intensivists need retrieve information with quality in which it will result in a more accurate and safer health care given to the patient.

The pervasive aspect of the timeline and the incorporation of data mining models are very important to improve the decision making process. In the first case it makes possible remote access to the timeline content at any moment, and the second because it makes future predictions over the patients' condition, allowing the intensivists to act in advance. Together, the artefact developed allows a more pro-active approach in the patients' best interest.

In the big scope, the Pervasive Patient Timeline allows the intensivists to acquire new lines of thought that lead them to consider relationships between the data that otherwise they would not consider.

\section{Conclusion}

The development of the Pervasive Patient Timeline showed that a new and innovative way of presenting information can, not only facilitate the job of intensivists, but also increase the quality of care given to patients, through the reduction of medical errors associated to the difficulty in crossing too much information. It covers a new approach in visualizing information which can be adopted in other similar situations. This flexibility allows for an easy addition of new data sources or data mining models in order to increase the amount of data presented on it, and therefore improve the quality of decisions made.

Its pervasiveness allows it to be accessed anytime and anywhere, through various and different devices. This option allows a simpler, accessible and functional access to the data and knowledge provided by the INTCare system.

This work proved the viability of adopting timelines as a way of visualizing clinical data of patients. It highlights the importance of a good and pleasant representation and presentation of the data, which can affect positively the decision of 
the intensivists, but also the importance of having only one platform that aggregates, homogenizes and displays distinct data, allowing for a faster, easier and intuitive access. Intensivists are also motivated to use the solution developed and its features, particularly the presentation of all patient's data sorted chronologically. Other positive aspect is the possibility of aggregating data from different data sources in the same location and the ability to access its information anywhere and anytime when they need to make decisions.

This new approach is important for Intensive Medicine, an environment with many technological devices that are constantly collecting and processing big amounts of data in real-time. All the collected data (with a date associated) can be incorporated into the Pervasive Patient Timeline in order to help the intensivists to make better and faster decisions in the patient's best interest. In the future some new models and data sources will be incorporated in the pervasive patient timeline. At same time the artefact developed will be also evaluated by the users (intensivists).

\section{Acknowledgements}

This work has been supported by FCT - Fundação para a Ciência e Tecnologia within the Project Scope UID/CEC/00319/2013. The authors would like to thank FCT for the financial support through the contract PTDC/EEI-SII/1302/2012 (INTCare II).

\section{References}

1. Direção Geral de Saúde, "Cuidados Intensivos: Recomendações para o seu desenvolvimento," D. G. d. Saúde, Ed., ed. Lisboa, (2003).

2. R. Haux, E. Ammenwerth, A. Winter, and B. Brigl, Strategic information management in hospitals: an introduction to hospital information systems: Springer, (2004).

3. A. Morris and A. Gardner, "Computer Applications," in Principles of Critical Care, J. Hall, G. Schmidt, and L. Wood, Eds., ed New York: McGraw-Hill, 1992, pp. 500-514 (1992).

4. A. Silva, "Modelos de intelegência artificial na análise da monitorização de eventos clínicos adversos, disfusão/falência de orgãos e prognóstico do doente crítico," (2007).

5. P. Suter, A. Armaganidis, F. Beaufils, X. Bonfill, H. Burchardi, D. Cook, et al., "Predicting outcome in ICU patients," Intensive Care Medicine, vol. 20, pp. 390-397, (1994).

6. F. Portela, M. Santos, J. Machado, A. Abelha, Á. Silva, and F. Rua, "Pervasive and Intelligent Decision Support in Intensive Medicine - The Complete Picture," in Information Technology in Bio- and Medical Informatics. vol. 8649, ed: Springer International Publishing, 2014, pp. 87-102 (2014).

7. J. Ramon, D. Fierens, F. Güiza, G. Meyfroidt, H. Blockeel, M. Bruynooghe, et al., "Mining data from intensive care patients," Advanced Engineering Informatics, vol. 21, pp. 243-256, (2007).

8. Filipe Portela, Manuel Filipe Santos, José Machado, Álvaro Silva and António Abelha. Pervasive and Intelligent Decision Support in Critical Health Care using Ensemble. Lecture Notes in Computer Science (LNCS) Volume 8060, 2013, pp 1-16. ISBN: 978-3-64240093-3. Springer. (2013).

9. Filipe Portela, Manuel Filipe Santos, Álvaro Silva, António Abelha, José Machado. Pervasive Ensemble Data Mining Models to Predict Organ Failure and Patient Outcome in 
Intensive Medicine. Communications in Computer and Information Science. Volume 415, 2013, pp 410-425. Springer. (2013).

10. R. Veloso, F. Portela, M. Santos, J. M. Machado, A. Abelha, Á. Silva, et al., "Real-time data mining models for predicting length of stay in intensive care units," in KMIS. (2014.

11. P. Braga, F. Portela, M. F. Santos, and F. Rua, "Data Mining Models to Predict Patient's Readmission in Intensive Care Units," presented at the ICAART, (2014).

12. D. Rosenberg and A. Grafton, Cartographies of time: A history of the timeline: Princeton Architectural Press, (2010).

13. C. McDonald and R. Gardner, "Computerized Management of Intensive Care Patients," in Images, Signals and Devices, ed: Springer, 1987, pp. 31-45. (1987)

14. M. Richardson, W. Reilly, and J. Kuntz. (2008, December 8). How Timeglider Works. Available: http://timeglider.com/how it works (2008)

15. D. J. Cook and S. K. Das, "How smart are our environments? An updated look at the state of the art," Pervasive and mobile computing, vol. 3, pp. 53--73, (2007).

16. D. J. Cook and S. K. Das, "Pervasive computing at scale: Transforming the state of the art," Pervasive and Mobile Computing, vol. 8, pp. 22--35, (2012).

17. Santos, M.F., Portela, F., Vilas-Boas, M., Machado, J., Abelha, A., Neves, J., Silva, A., Rua, F, "A pervasive approach to a real-time intelligent decision support system in intensive medicine," pp. 368--381, (2012).

18. U. Varshney, "Pervasive Computing," in Pervasive Healthcare Computing, ed: Springer US, (2009).

19. K. Peffers, T. Tuunanen, M. A. Rothenberger, and S. Chatterjee, "A design science research methodology for information systems research," Journal of management information systems, vol. 24, pp. 45-77 (2007)

20. André Braga, Filipe Portela, Manuel Filipe Santos, José Machado, António Abelha, Álvaro Silva and Fernando Rua. Data Mining to predict the use of Vasopressors in Intensive Medicine Patients. Jurnal Teknologi. (2016).

21. Filipe Portela, Manuel Filipe Santos, António Abelha, José Machado, Fernando Rua Martins and Álvaro Silva. Real-time Decision Support using Data Mining to predict Blood Pressure Critical Events in Intensive Medicine Patients. LNCS - Ambient Intelligence for Health. Volume 9456, 2015, pp 77-90. Springer. (2015)

22. Filipe Portela, Manuel Santos, Jose Machado, Antonio Abelha, Álvaro Silva, Fernando Rua. Preventing Patient Cardiac Arrhythmias by Using Data Mining Techniques. IECBES 2014 - 2014 IEEE. (2015).

23. Filipe Portela, Rui Veloso, Sérgio Oliveira, Manuel Filipe Santos, António Abelha, José Machado, Álvaro Silva and Fernando Rua. Predict hourly patient discharge probability in Intensive Care Units using Data Mining. Indian Journal of Science and Technology. Indian Society for Educat. (2016).

24. João M. C. Gonçalves, Filipe Portela, Manuel F. Santos, Álvaro Silva, José Machado, António Abelha, Fernando Rua. Real-time Predictive Analytics for Sepsis Level and Therapeutic Plans in Intensive Care Medicine. IJHISI. Volume 9, Issue 3, pp 36-54. IGI Global. (2014) 